\title{
Dinámicas relacionales entre investigadores: una perspectiva desde el análisis de redes sociales
}

\author{
Relational Dynamics Between Researchers: A Social Network Analysis \\ Perspective
}

\section{Dinâmica relacional entre pesquisadores: uma perspectiva a partir da análise de redes sociais}

\author{
Ángel Eustorgio Rivera González \\ Instituto Politécnico Nacional, México \\ aerivera@ipn.mx \\ https://orcid.org/0000-0001-5636-9825 \\ Claudia Alejandra Hernández Herrera \\ Instituto Politécnico Nacional, México \\ cahernandezh@ipn.mx \\ https://orcid.org/0000-0002-4060-2941
}

\section{Resumen}

El objetivo de este trabajo de investigación es entender el funcionamiento de una red social integrada por profesores-investigadores de un departamento de posgrado de una universidad mexicana. Los actores analizados participan en diversas actividades académicas y de investigación, tales como dirigir o ser sinodales de tesis de maestría. Este estudio comprende, en primera instancia, el análisis de las relaciones laborales que establecen los profesores con base en su participación en proyectos de tesis, y por otro lado, se analiza la fuerza de sus lazos sociales, así como las relaciones de ayuda y apoyo que se brindan entre ellos. La perspectiva teórico metodológica para analizar las relaciones entre los actores se basa en la teoría de redes sociales. Los resultados muestran que existe una fragmentación del grupo de investigadores y que, en consecuencia, existen varios grupos de estos que determinan una 

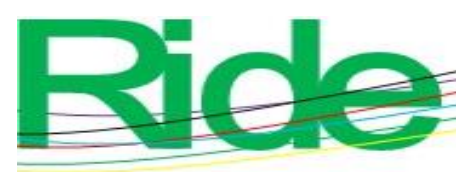

Revista Iberoamericana para la Investigación y el Desarrollo Educativo ISSN $2007-7467$

estructura informal distinta a la formalmente establecida. Esta estructura informal también guía el desarrollo de las actividades de investigación en el departamento. Los hallazgos revelan que existen actores estratégicos o claves en la red y, por tanto, los miembros en general no participan de manera homogénea ni equitativa en la conducción de los trabajos de tesis de los alumnos.

Palabras clave: canales de comunicación, colaboración científica, relaciones laborales, transferencia de conocimiento científico, universidad.

\section{Abstract}

The objective of this research is to analyze as well as to understand the performance of a social network integrated by researchers of a postgraduate department of a Mexican public university. The actors that are analyzed in this research actively participate in thesis projects (advisors and examiners). This study includes, on first place, the analysis of labor relationships established by professors based on their experiences as collaborators in thesisprojects. On second place, this study examines the strength of professors' social relationships (ties), as well as their mutual support and assistance relationships. This study is based on the social network analysis approach. Our findings show that there is a clusterization in the researchers' group and as a consequence exist several groups of researchers that determine an informal structure different from the defined formal structure. The results indicate strong support for the fact that the informal structure guides the development of the research activities of the department. Data reveals that strategic or key actors exist and, consequently, members do not participate in homogenous nor equitable way in the development of academic work.

Keywords: communication channels, scientific collaboration, labor relationships, scientific knowledge transfer, university. 


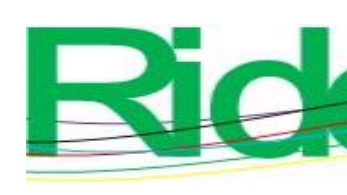

Revista Iberoamericana para la Investigación y el Desarrollo Educativo ISSN $2007-7467$

\section{Resumo}

O objetivo deste trabalho de pesquisa é compreender o funcionamento de uma rede social composta por professores-pesquisadores de um departamento de pós-graduação de uma universidade mexicana. Os atores analisados participam de diversas atividades acadêmicas e de pesquisa, como dirigir ou serem sínodos das teses de mestrado. Este estudo inclui, em um primeiro momento, a análise das relações de trabalho estabelecidas pelos professores com base em sua participação em projetos de tese e, por outro lado, a força de seus laços sociais, bem como as relações de ajuda e apoio. que se oferecem. A perspectiva metodológica teórica para analisar as relações entre os atores é baseada na teoria das redes sociais. Os resultados mostram que há uma fragmentação do grupo de pesquisadores e, consequentemente, existem vários grupos de pesquisadores que determinam uma estrutura informal diferente da formalmente estabelecida. Essa estrutura informal também orienta o desenvolvimento de atividades de pesquisa no departamento. Os resultados revelam que existem atores estratégicos ou chave na rede e, portanto, os membros em geral não participam de maneira homogênea ou equitativa na condução do trabalho de tese dos alunos.

Palavras-chave: canais de comunicação, colaboração científica, relações de trabalho, transferência de conhecimento científico, universidade.

Fecha Recepción: Febrero 2020

Fecha Aceptación: Julio 2020

\section{Introducción}

Las interacciones entre individuos representan un elemento esencial de los grupos sociales. El conocimiento, por su parte, es inherente al ser humano. Entonces, el estudio y análisis de la transferencia del conocimiento y las implicaciones que esta transferencia tiene en los grupos sociales toma gran relevancia. En términos generales, y para el estudio de las organizaciones, el conocimiento se crea a través de interacciones humanas (De Long y Fahey, 2000; Nonaka y Takeuchi, 1995; Tasselli, Kilduff y Menges, 2015; Wuebker, Hampl y Wüstenhagen, 2015), es útil para la acción (Davenport y Prusak, 1998; De Long y Fahey, 2000; Hjorth, Holt y Steyaert, 2015; Probst, Raub y Romhardt 2000; Quinn, Anderson y Finkelstein, 1996) y está representado por un conjunto de habilidades, creencias, experiencias e intuición (Davenport y Prusak, 1998; De Long y Fahey, 2000; Kerssens, De Weerd y Fisscher, 1996; Sankar, Asokan y Satheesh, 2015). 


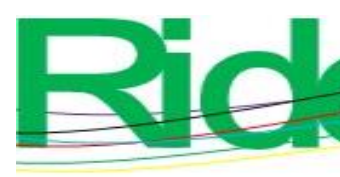

Revista Iberoamericana para la Investigación y el Desarrollo Educativo ISSN $2007-7467$

ingresó al Programa Nacional de Posgrados de Calidad (PNPC) del Consejo Nacional de Ciencia y Tecnología (Conacyt), y se habla en los pasillos sobre proyectos de investigación.

Desde esta perspectiva, y desde el punto de vista de los investigadores, el grupo de profesores-investigadores que existe ha creado una estructura informal de organización que dista de la estructura formal definida por la institución. Los profesores del departamento analizado están integrados de manera formal en tres distintos grupos de trabajo, que responden a la naturaleza y estructura formal del departamento. Dentro del departamento existen tres distintos programas de posgrado (denominados $P-1, P-2, P-3$ ) y los profesoresinvestigadores se encuentran adscritos a uno o más de estos programas académicos dependiendo de sus áreas de interés y su experiencia profesional. Los actores de esta red se encuentran distribuidos en dos de los tres pisos del edificio del departamento.

\section{Materiales y métodos}

En esta investigación se realizaron observaciones etnográficas, se aplicaron encuestas a los participantes y se consultaron fuentes secundarias como documentos oficiales y las bases de datos del sistema de información interno de la institución. Parte del análisis que se realiza en este trabajo de investigación se basa en observaciones etnográficas de dos de los profesores investigadores que participan en los programas de maestría del departamento analizado.

Para poder describir las relaciones existentes entre los actores sociales de este estudio, se analizaron los datos de archivo con respecto a los proyectos de desarrollo de tesis de grado dentro del departamento. Se analizó la participación de los profesores en cada uno de los comités de tesis durante un periodo de nueve años (del 2000 al 2009). Los datos que respaldan esta información fueron recabados de los registros (tanto en formato electrónico como en documentos impresos) de la institución. Adicionalmente, se aplicó una encuesta a los actores sociales cuyo objetivo fue recabar información que permita entender el funcionamiento de la estructura social de los participantes en la organización y de las funciones particulares de los actores, así como de las estrategias de transferencia y difusión del conocimiento. Para ello, las relaciones que existen entre los profesores-investigadores, así como las fuerzas de sus lazos de colaboración, fueron analizadas. La encuesta se dividió en dos secciones. En la primera de ellas se plantearon preguntas con el objetivo de obtener datos descriptivos de la muestra (miembros de la red social), y en la segunda sección, las preguntas se diseñaron para 


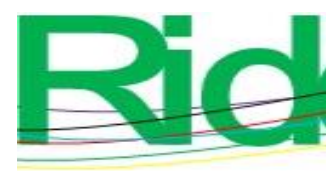

Revista Iberoamericana para la
Investigación y el Desarrollo Educativo
ISSN $2007-7467$

obtener información con respecto a las relaciones que existen entre los miembros de la red social.

Las encuestas fueron aplicadas de manera personal a los participantes de la red social en un periodo de dos semanas. La tasa total de respuesta fue de $68.96 \%$ (20/29). Para garantizar la confidencialidad de las respuestas y promover la sinceridad de estas, se juzgó conveniente que en la encuesta no se colocara el nombre del actor social que la respondiera. Para ello, se asignó un número consecutivo (1 al 29) a cada una de las encuestas.

Los actores sociales analizados en este trabajo de investigación son 29 profesoresinvestigadores asignados a un departamento que tiene tres áreas distintas. Respecto al nivel académico, $44.82 \%$ de los actores sociales tiene estudios de doctorado y el resto tiene estudios de maestría. En cuestiones de género, 5 de los 29 profesores son mujeres, lo que representa $17.24 \%$ de la muestra. Todos los profesores se encuentran distribuidos en dos pisos de un edificio y cada uno de ellos cuenta con oficina propia. La edad promedio de la muestra es de 51 años, y la antigüedad promedio de los miembros del departamento es de 13 años.

La distribución de los actores sociales dentro de los grupos formales e informales es fundamental para el desarrollo de este trabajo de investigación. De acuerdo con las fuentes secundarias consultadas y con las observaciones etnográficas realizadas, clasificamos a los actores sociales en cuatro subgrupos fundamentalmente (tabla 1). Los subgrupos los identificamos con las letras $A, B, C$ y $D$. Cabe mencionar que en este trabajo se recurrió al software Ucinet para el análisis de las redes sociales. 


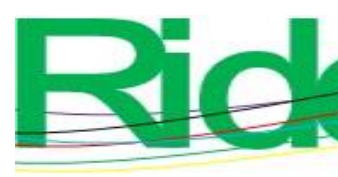

Revista Iberoamericana para la Investigación y el Desarrollo Educativo ISSN $2007-7467$

Tabla 1. Afiliaciones formales e informales de los actores sociales

\begin{tabular}{|l|l|l|}
\hline & & \multicolumn{1}{c|}{ Actores (claves) } \\
\hline & P-1 & $\begin{array}{l}\text { A-1, A-4, A-5, A-6, A-7, A-9, A-10, A-11, A-12, A-16, A-19, A-20. A-21, A- } \\
22, \text { A-25. A-27, A-28, A-29. }\end{array}$ \\
\hline A & P-2 & $\begin{array}{l}\text { A-6, A-9, A-12, A-13, A-14, A-15, A-17, A-18, A-20, A-21, A-22, A-23, A-24, } \\
\text { A-26, A-28 }\end{array}$ \\
\hline & P-3 & A-1, A-2, A-3, A-5, A-8, A-15, A-16, A-17, A-19, A-20, A-27, A-29 \\
\hline & P-1 & $\begin{array}{l}\text { A-4, A-5, A-6, A-7, A-9, A-10, A-11, A-12, A-14, A-16, A-19, A-20, A-25, A- } \\
27, \text { A-28, A-29 }\end{array}$ \\
\hline C & P-2 & $\begin{array}{l}\text { A-6, A-9, A-11, A-12, A-13, A-15, A-17, A-18, A-20, A-21, A-22, A-23, A-24, } \\
\text { A-26 }\end{array}$ \\
\hline & P-3 & A-1, A-2, A-3, A-5, A-8, A-16, A-29 \\
\hline & G-1 & A-3, A-4, A-5, A-9, A-10, A-16, A-19, A-27 \\
\hline B & G-2 & A-7, A-11, A-12, A-14, A-20, A-23, A-24, A-26, A-29 \\
\hline & G-3 & A-6, A-15, A-18, A-18, A-21, A-22, A-28 \\
\hline & G-4 & A-1, A-2, A-8, A-15, A-17, A-18, A-21, A-22, A-27, A-28 \\
\hline D & G-a & $\begin{array}{l}\text { A-1, A-2, A-4, A-5, A-7, A-8, A-9, A-10, A-11, A-12, A-13, A-14, A-16, A-19, } \\
\text { A-20, A-23, A-24, A-25, A-26, A-29 }\end{array}$ \\
\hline & G-b & A-3, A-6, A-15, A-17, A-18, A-21, A-22, A-27, A-28 \\
\hline
\end{tabular}

$A=$ Distribución oficial; $C=$ Real y tradición; $B=$ Grupo de afiliación; $D=$ Hegemónico y no hegemónico.

$P-1=$ Programa $1 ; P-2=$ Programa $2 ; P-3=$ Programa $3 ; G-1=$ Grupo $1 ; G-2=$ Grupo 2;

$$
G-3=\text { Grupo 3; } G-4=\text { Grupo } 4 .
$$

$G$ - $a$ = Grupo hegemónico; $G-b=$ Grupo no hegemónico .

Fuente: Elaboración propia

Los componentes definidos como $A$ y $C$ representan la estructura formal de la organización. El primero de ellos (A) representa los núcleos académicos en los cuales están divididos los actores sociales dentro del departamento. Es importante mencionar que en esta clasificación un actor social puede pertenecer a uno o más de los tres programas en lo que se divide el departamento. El componente $\mathrm{C}$ se refiere a la manera en cómo los actores desempeñan sus actividades formales con respecto a la asistencia a reuniones y toma de decisiones en ellas. Este componente es la manera tradicional de funcionamiento del 

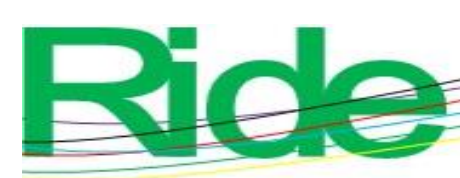

Revista Iberoamericana para la Investigación y el Desarrollo Educativo ISSN $2007-7467$

de tesis de grado como sinodal; $j$ ) Tesis totales por actor social, en donde cada celda de la matriz representa la diferencia absoluta entre las participaciones de un actor $i$ con respecto a uno $j$ en el desarrollo de tesis de grado como director; $k$ ) Total director porcentaje, en donde cada celda de la matriz (dicotomizada mayor de $25 \%$ de participación conjunta) representa si un actor $i$ tuvo la misma intervención que un actor $j$ (mayor de $25 \%$ de participación conjunta) en la dirección de tesis de grado; l) Total director sinodal porcentaje, en donde cada celda de la matriz (dicotomizada - mayor de $25 \%$ de participación conjunta) indica si un actor $i$ tuvo la misma intervención que un actor $j$ en la dirección y sinodalías de tesis de grado; m) Hegemónico o no hegemónico, en donde cada celda de la matriz representa la coincidencia o no de un actor $i$ con un actor $j$ en un grupo hegemónico o no hegemónico determinado por observación etnográfica; $n$ ) Distribución oficial, en donde cada celda representa si un actor $i$ se encuentra en al menos un núcleo académico definido formalmente por el departamento similar al del actor $j ; \tilde{n}$ ) Real y tradición, en donde cada celda de la matriz representa si un actor $i$ se encuentra en al menos un programa (P-1, P-2, P-3) definido formalmente por el departamento similar al del actor $j$.

La información obtenida de las fuentes secundarias y las respuestas de las encuestas contestadas se procesaron en tablas de Microsoft Office Excel para facilitar su manejo. Se diseñaron tablas y matrices distintas que contienen la información relevante del estudio. En el análisis de gran parte de la información obtenida en este trabajo de investigación se utilizó el enfoque de redes sociales, ya que ofrece una perspectiva amplia de las relaciones o lazos que se establecen entre los miembros del departamento. Para analizar los datos estadísticamente, utilizamos correlaciones. Debido a que las observaciones obtenidas a través del análisis de redes sociales no son independientes, no satisfacen los supuestos de la estadística inferencial. 

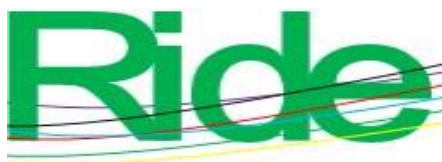

Revista Iberoamericana para la Investigación y el Desarrollo Educativo

ISSN $2007-7467$

\section{Resultados}

En el desarrollo de esta sección del artículo se consideró prudente realizar nuestro análisis basándonos en dos aspectos fundamentales que distinguimos en la interacción de los actores sociales bajo análisis:

1) La existencia de una estructura informal que es distinta a la estructura formalmente establecida. Esta estructura informal guía el desarrollo de las actividades de investigación en el departamento analizado.

2) La existencia de actores claves que guían el desarrollo de las actividades académicas del departamento (desarrollo de proyectos de investigación y dirección de tesis de grado).

De nuestro estudio exploratorio podemos argumentar los siguientes resultados generales con respecto a las correlaciones obtenidas en esta investigación (tabla 2). 


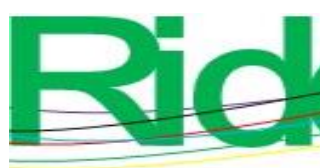

Revista Iberoamericana para la Investigación y el Desarrollo Educativo ISSN 2007 - 7467

Tabla 2. Correlaciones. Paradigma de asignación cuadrática (QAP). Generales

\begin{tabular}{|c|c|c|c|c|c|c|c|c|c|c|c|c|c|}
\hline & $\mathbf{A}$ & B & C & D & $\mathbf{E}$ & $\mathbf{F}$ & $\mathbf{G}$ & $\mathbf{H}$ & I & $\mathbf{J}$ & $\mathbf{K}$ & $\mathbf{L}$ & $\mathbf{M}$ \\
\hline $\mathbf{A}$ & 1.00 & & & & & & & & & & & & \\
\hline B & 0.14 & 1.00 & & & & & & & & & & & \\
\hline $\mathrm{C}$ & $\begin{array}{l}0.15 \\
* *\end{array}$ & -0.02 & 1.00 & & & & & & & & & & \\
\hline D & $\begin{array}{l}0.18 \\
*\end{array}$ & $\begin{array}{l}0.12 \\
*\end{array}$ & $\begin{array}{l}0.09 \\
*\end{array}$ & 1.00 & & & & & & & & & \\
\hline $\mathbf{E}$ & -0.03 & 0.00 & 0.03 & -0.01 & 1.00 & & & & & & & & \\
\hline $\mathbf{F}$ & -0.02 & -0.04 & -0.02 & -0.08 & -0.03 & 1.00 & & & & & & & \\
\hline $\mathbf{G}$ & $\begin{array}{l}0.22 \\
*\end{array}$ & $\begin{array}{l}0.29 \\
* *\end{array}$ & 0.06 & $\begin{array}{l}0.17 \\
*\end{array}$ & 0.00 & -0.04 & 1.00 & & & & & & \\
\hline $\mathbf{H}$ & $\begin{array}{l}-0.17 \\
* *\end{array}$ & 0.01 & $\begin{array}{l}-0.97 \\
* *\end{array}$ & -0.08 & -0.03 & 0.03 & $\begin{array}{l}-0.09 \\
*\end{array}$ & 1.00 & & & & & \\
\hline I & $\begin{array}{l}0.24 \\
*\end{array}$ & $\begin{array}{l}0.31 \\
* *\end{array}$ & 0.05 & $\begin{array}{l}0.17 \\
*\end{array}$ & 0.00 & -0.05 & $\begin{array}{l}0.99 \\
* *\end{array}$ & $\begin{array}{l}-0.08 \\
*\end{array}$ & 1.00 & & & & \\
\hline $\mathbf{J}$ & 0.16 & $\begin{array}{l}0.19 \\
*\end{array}$ & $\begin{array}{l}0.09 \\
*\end{array}$ & 0.12 & 0.01 & 0.00 & $\begin{array}{l}0.87 \\
* *\end{array}$ & $\begin{array}{l}-0.12 \\
*\end{array}$ & $\begin{array}{l}0.81 \\
* *\end{array}$ & 1.00 & & & \\
\hline $\mathbf{K}$ & $\begin{array}{l}-0.13 \\
*\end{array}$ & -0.05 & -0.01 & $\begin{array}{l}-0.10 \\
*\end{array}$ & -0.02 & $\begin{array}{l}0.13 \\
* *\end{array}$ & 0.09 & 0.00 & 0.10 & 0.05 & 1.00 & & \\
\hline $\mathbf{L}$ & -0.10 & -0.03 & $\begin{array}{l}-0.07 \\
*\end{array}$ & -0.01 & -0.03 & $\begin{array}{l}0.09 \\
*\end{array}$ & $\begin{array}{l}0.28 \\
* *\end{array}$ & 0.05 & $\begin{array}{l}0.29 \\
* *\end{array}$ & $\begin{array}{l}0.22 \\
*\end{array}$ & $\begin{array}{l}0.55 \\
* *\end{array}$ & 1.00 & \\
\hline $\mathbf{M}$ & 0.00 & 0.05 & $\begin{array}{l}-0.13 \\
*\end{array}$ & $\begin{array}{l}-0.31 \\
* *\end{array}$ & -0.02 & $\begin{array}{l}0.35 \\
* *\end{array}$ & 0.03 & $\begin{array}{l}0.11 \\
*\end{array}$ & 0.03 & 0.04 & $\begin{array}{l}0.18 \\
* *\end{array}$ & $\begin{array}{l}0.13 \\
*\end{array}$ & 1.00 \\
\hline
\end{tabular}

$*=p<0.05, * *=p<0.01$

$A=$ Antigüedad en departamento; $B=$ Clases impartidas; $C=$ Distancia en metros; $D=$

Edad; $E=$ Grado de estudios; $F=$ Grupo de afiliación; $G=$ Participación total por actor social; $H=$ Piso de ubicación; $I=$ Sinodalías totales por actor social; $J=$ Tesis totales por actor social; $K=$ Total director porcentaje; $L=$ Total director sinodal porcentaje; $M=$

Hegemónico - No hegemónico

Fuente: Elaboración propia 


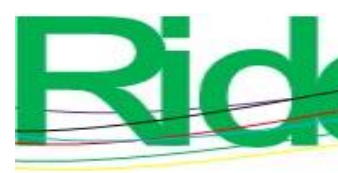

e

Revista Iberoamericana para la Investigación y el Desarrollo Educativo ISSN $2007-7467$

Las correlaciones sugieren que las estructuras formales (A y C) se encuentran correlacionadas de manera significativa $(p<0.01)$ con un índice de 0.28 , mientras que las estructuras informales (B y D) también se encuentran correlacionadas de manera significativa $(p<0.01)$ con un índice de 0.35 .

La correlación que existen entre los componentes formales e informales (A y B; A y D; B y C; C y D) son menores a las que existen en el análisis de las estructuras formales entre ellas y a las que existen en el análisis de las estructuras informales entre ellas. Incluso en algunos casos estas correlaciones no son significativas. Debido a la poca o nula correlación que existe entre los componentes formales e informales podemos argumentar que las estructuras difieren en sus arreglos y elementos, es decir, aunque exista una definición de estructuras formales en el departamento, estas estructuras se ven rebasadas por las relaciones que se establecen por los integrantes de los grupos informales.

La tabla 4 muestra los valores de densidad de los grupos existentes dentro del departamento. Para el cálculo de estas densidades se dicotomizó la matriz de la participación de los actores sociales como directores de tesis y sus colaboraciones con otros actores como sinodales (mayor de $25 \%$ de participación conjunta).

Tabla 4. Densidades de los grupos de afiliación

\begin{tabular}{|l|c|c|c|c|c|c|c|}
\hline & General & G-1 & G-2 & G-3 & G-4 & Hegemónico & $\begin{array}{c}\text { No } \\
\text { hegemónico }\end{array}$ \\
\hline Densidad & 0.1096 & 0.2679 & 0.1389 & 0.0952 & 0.05 & 0.1684 & 0.0694 \\
\hline
\end{tabular}

Fuente: Elaboración propia

Los resultados revelan que la densidad de G-1 es al menos dos veces la densidad de los demás grupos existentes en el departamento. Esto implica que la relación entre los miembros de este grupo (G-1) es mucho más frecuente que aquella que se presenta entre los integrantes al interior de los otros grupos del departamento.

Para detectar a los actores claves de la red utilizamos los valores que obtuvimos de las relaciones existentes entre los participantes de los proyectos de tesis. 


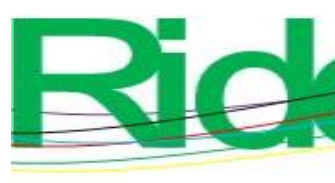

Revista Iberoamericana para la Investigación y el Desarrollo Educativo ISSN 2007 - 7467

Tabla 5. Actores sociales. Directores y sinodales de tesis de grado

\begin{tabular}{|c|c|c|c|c|c|c|c|}
\hline Actor & $\begin{array}{l}\text { Director } \\
\text { de tesis }\end{array}$ & $\begin{array}{l}\text { Sinodal } \\
\text { de tesis }\end{array}$ & $\begin{array}{c}\text { Total } \\
\text { (director } \\
+ \\
\text { sinodal) }\end{array}$ & Actor & $\begin{array}{l}\text { Director } \\
\text { de tesis }\end{array}$ & $\begin{array}{l}\text { Sinodal } \\
\text { de tesis }\end{array}$ & $\begin{array}{c}\text { Total } \\
\text { (director } \\
+ \\
\text { sinodal) }\end{array}$ \\
\hline A-1 & 0 & 1 & 1 & A-16 & 5 & 35 & 40 \\
\hline A-2 & 3 & 25 & 28 & A-17 & 0 & 2 & 2 \\
\hline A-3 & 2 & 3 & 5 & A-18 & 3 & 7 & 10 \\
\hline A-4 & 0 & 21 & 21 & A-19 & 14 & 82 & 96 \\
\hline A-5 & 23 & 78 & 101 & A-20 & 6 & 12 & 18 \\
\hline A-6 & 12 & 15 & 27 & A-21 & 0 & 5 & 5 \\
\hline A-7 & 6 & 11 & 17 & A-22 & 0 & 6 & 6 \\
\hline A-8 & 0 & 0 & 0 & A-23 & 2 & 13 & 15 \\
\hline A-9 & 13 & 48 & 61 & A-24 & 0 & 2 & 2 \\
\hline A-10 & 16 & 50 & 66 & A-25 & 7 & 9 & 16 \\
\hline A-11 & 0 & 0 & 0 & A-26 & 4 & 6 & 10 \\
\hline A-12 & 7 & 34 & 41 & A-27 & 0 & 0 & 0 \\
\hline A-13 & 7 & 22 & 29 & A-28 & 0 & 0 & 0 \\
\hline A-14 & 0 & 9 & 9 & A-29 & 22 & 81 & 103 \\
\hline A-15 & 1 & 3 & 4 & & & & \\
\hline
\end{tabular}

Promedio de tesis dirigidas $\mathrm{x}$ actor: 5; Promedio de sinodalías de tesis $\mathrm{x}$ actor: 20;

Promedio del total (director + sinodal) x actor: 25 .

Fuente: Elaboración propia

Del análisis de los datos de la tabla 5 se puede distinguir que 12 actores se encuentran por arriba de la media en cuanto a la dirección de trabajos de tesis. De estos 12 actores, son 6 lo que rebasan en 10 el número de tesis dirigidas en el periodo analizado (A-5, A-6, A-9, A-10, A-19, A-29). Es importante señalar que todos estos actores de la red social (excepto A-6) pertenecen al grupo hegemónico informal (G-a).

También es importante analizar, por otro lado, la participación en sinodalías de tesis de grado. En este sentido, los actores con más participación en sinodalías de tesis son A-2, A-4, A-5, A-9, A-10, A-12, A-13, A-16, A-19, A-29, todos estos actores sociales también pertenecen al grupo hegemónico informal (G-a). De los 153 trabajos de tesis analizados 


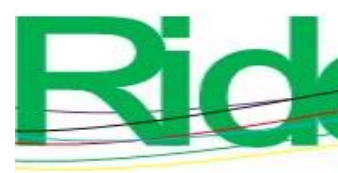

Revista Iberoamericana para la Investigación y el Desarrollo Educativo ISSN $2007-7467$

durante el periodo 2000-2009, 65.35\% (100/153) han sido dirigidas por 6 de 29 actores sociales de la red. De acuerdo con la tabla 2, existe una correlación moderada (-0.13) y significativa $(p<0.05)$ de que los actores sociales con mayor tiempo en el departamento son los actores que más tesis dirigen.

Los datos analizados revelan que los miembros de la red social no participan de manera homogénea en el desarrollo de trabajos de tesis aun y cuando su participación es fomentada de la misma manera.

\section{Discusión}

En el estudio realizado hemos corroborado la importancia de las prácticas socioculturales de los actores sociales considerados en la red de análisis. El análisis de nuestros datos revela que no todos los miembros de la red participan de manera similar. De acuerdo con el análisis realizado, podemos argumentar que la comunicación dentro de la red y el proceso para que ella se desarrolle de manera efectiva depende de algunos actores en particular. A este efecto Neus (2001) lo denomina la visión del túnel de la comunicación.

Aunque los actores de la red social analizados comparten un dominio o interés común de conocimiento, es evidente que este interés es dependiente de algunos factores que giran en torno a cada elemento de la red. Alavi y Tiwana (2002) argumentan que entre estos factores se encuentran el clima y cultura organizacional, la distribución física, el tipo de trabajo realizado y el acceso a la información y tecnología.

El análisis revela que la forma en cómo se comparte y se distribuye la información dentro de la comunidad estudiada no es simétrica, ello implica la existencia de una clusterización de la red. En este trabajo se encontró evidencia de lo que se conoce como inflexibilidad en los lazos organizacionales (Hite y Hesterly, 2001; Hjorth et al., 2015; Shipilov, Gulati, Kilduff, Li y Tsai, 2014). Los lazos del equipo de trabajo son débiles ya que las interacciones son poco frecuentes (Hansen, 1999). Es importante mencionar que, de acuerdo con los datos obtenidos en este estudio, existe una diferencia significativa entre la participación de los integrantes de la red, aunque en teoría está participación se fomenta de manera similar en todos los integrantes. Las posiciones estratégicas que ocupan algunos elementos de la red son un detonante que promueve $\mathrm{y}$, en algunos casos, limita la participación activa de todos los actores sociales. Este efecto implica la existencia de una muy baja e incluso nula correlación entre los componentes formales e informales, y ello 


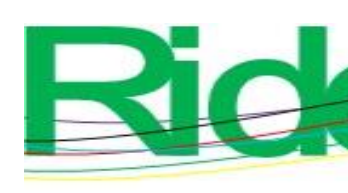

Revista Iberoamericana para la Investigación y el Desarrollo Educativo ISSN $2007-7467$

implica que las estructuras difieran en sus arreglos y elementos. El desarrollo de las prácticas académicas de los diferentes actores sociales definidas formalmente en las estructuras de la organización son distintas a las prácticas que se desarrollan en la cotidianeidad de las actividades organizacionales.

Finalmente, es importante destacar que en la realización del presente estudio se identificó la existencia de hábitos y prácticas defensivas por parte de algunos actores que impiden realizar un análisis completo de la estructura social (informal) de la organización.

\section{Conclusiones}

Se concluye que el artículo cumplió su objetivo, que fue el entendimiento del funcionamiento de una red social integrada por profesores investigadores de una sección de estudios de posgrado de una universidad en Ciudad de México. Las implicaciones para las instituciones de educación superior son diversas. En principio, un análisis de este tipo permite entender las dinámicas que se gestan en las redes sociales que se tienen en las organizaciones académicas, estas tienen su origen en grupos formales o en grupos informales. Sumado a lo anterior, permite razonar las dinámicas de las relaciones entre los grupos que se encuentran en departamentos que desarrollan investigación, en este caso en el área de las ciencias sociales. Sin embargo, es una realidad que existen grupos que afianzan privilegios dentro de las estructuras y las redes y que, por ello, obtienen más beneficios, como es el caso de la dirección de los trabajos de tesis, por solo mencionar uno, por lo que es relevante el estudio de los grupos que se gestan para entender la forma en la que ellos dictan las formas del trabajo académico.

Las limitaciones que se tuvieron en el desarrollo de la investigación fueron las prácticas a la defensiva que traban el proceso de obtención y posterior análisis de la información, aunado a la escasa presencia de variables que hubieran permitido entender a profundidad el fenómeno como la disciplina, el prestigio del académico, la habilitación científica, el carisma, la amistad, los vínculos afectivos y el peso político de los actores en círculos de toma de decisión en las instituciones de educación superior. Las ventajas de este tipo de investigación es que apoyan en el entendimiento de los grupos de trabajo y la forma en cómo establecen acuerdos de apoyo mutuo para alcanzar metas en la generación de conocimiento. 


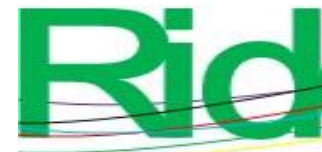

Information Science Research, 30(4), 282-290. Retrieved from https://doi.org/10.1016/j.lisr.2008.04.003.

Tasselli, S., Kilduff, M. and Menges, J. (2015). The Microfoundations of Organizational Social Networks: A Review and an Agenda for Future Research. Journal of Management, 41(5), 1361-1387. Retrieved from https://doi.org/10.1177/0149206315573996.

Tierney, W. (1988). Organizational culture in higher education: defining the essentials. Journal of Higher Education, 59(1), 2-21. Retrieved from https://doi.org/10.1080/00221546.1988.11778301.

Wasserman, S. and Faust K. (1994). Structural Analysis in the Social Science - Social Networks Analysis - Methods and Applications. California, United States: SAGE Publications.

Wuebker, R., Hampl, N. and Wüstenhagen, R. (2015). The Strength of Strong Ties in an Emerging Industry: Experimental Evidence of the Effects of Status Hierarchies and Personal Ties in Venture Capitalist Decision Making. Strategic Entrepreneurship Journal, 9(2), 167-187. Retrieved from https://doi.org/10.1002/sej.1188.

Zhang, S. and Liu, J. (2007). Autonomy-oriented social networks modeling: Discovering the dynamics of emergent structure and performance. International Journal of Pattern Recognition and Artificial Intelligence, 21(4), 611-638. Retrieved from https://doi.org/10.1142/S0218001407005582. 


\begin{tabular}{|l|l|}
\hline Rol de Contribución & Autor (es) \\
\hline Conceptualización & Ángel Eustorgio Rivera González (principal) \\
\hline Metodología & $\begin{array}{l}\text { Ángel Eustorgio Rivera González (igual) } \\
\text { Claudia Alejandra Hernández Herrera (igual) }\end{array}$ \\
\hline Software & $\begin{array}{l}\text { Ángel Eustorgio Rivera González (igual) } \\
\text { Claudia Alejandra Hernández Herrera (igual) }\end{array}$ \\
\hline Validación & Ángel Eustorgio Rivera González (principal) \\
\hline Análisis Formal & $\begin{array}{l}\text { Ángel Eustorgio Rivera González (principal) } \\
\text { Claudia Alejandra Hernández Herrera (apoyo) }\end{array}$ \\
\hline Investigación & $\begin{array}{l}\text { Ángel Eustorgio Rivera González (principal) } \\
\text { Claudia Alejandra Hernández Herrera (apoyo) }\end{array}$ \\
\hline Recursos & $\begin{array}{l}\text { Ángel Eustorgio Rivera González (principal) } \\
\text { Claudia Alejandra Hernández Herrera }\end{array}$ \\
\hline Curación de datos & $\begin{array}{l}\text { Ángel Eustorgio Rivera González (igual) } \\
\text { Claudia Alejandra Hernández Herrera (igual) }\end{array}$ \\
\hline $\begin{array}{l}\text { Escritura - Preparación del } \\
\text { borrador original }\end{array}$ & Ángel Eustorgio Rivera González (principal) \\
\hline $\begin{array}{l}\text { Escritura - Revisión y } \\
\text { edición }\end{array}$ & $\begin{array}{l}\text { Ángel Eustorgio Rivera González (igual) } \\
\text { Claudia Alejandra Hernández Herrera (igual) }\end{array}$ \\
\hline Visualización & $\begin{array}{l}\text { Ángel Eustorgio Rivera González (igual) } \\
\text { Claudia Alejandra Hernández Herrera (igual) }\end{array}$ \\
\hline Supervisión & $\begin{array}{l}\text { Ángel Eustorgio Rivera González (principal) } \\
\text { Claudia Alejandra Hernández Herrera (apoya) }\end{array}$ \\
\hline $\begin{array}{l}\text { Administración de } \\
\text { Proyectos }\end{array}$ & $\begin{array}{l}\text { Ángel Eustorgio Rivera González (igual) } \\
\text { Claudia Alejandra Hernández Herrera (igual) }\end{array}$ \\
\hline Adquisición de fondos & $\begin{array}{l}\text { Ángel Eustorgio Rivera González (igual) } \\
\text { Claudia Alejandra Hernández Herrera (igual) }\end{array}$ \\
\hline
\end{tabular}

